\title{
Effect of Haptic Assistance on Learning Vehicle Reverse Parking Skills
}

\author{
Masakazu Hirokawa, Student member, IEEE, Naohisa Uesugi, Satoru Furugori, Tomoko Kitagawa, \\ and Kenji Suzuki, Member, IEEE
}

\begin{abstract}
Compared to conventional visual- and auditory-based assisted driving technologies, haptic modality promises to be more effective and less disturbing assistance to the driver. However, in most previous studies, haptic assistance systems were evaluated from safety and stability viewpoints. Moreover, the effect of haptic assistance on human driving behavior has not been sufficiently discussed. In this paper, we introduce an assisted driving method based on haptic assistance for driver training in reverse parking, which is considered as an uncertain factor in conventional assisted driving systems. The proposed system assists the driver by applying a torque on the steering wheel to guide proper and well-timed steering. To design the appropriate assistance method, we conducted a measurement experiment to determine the qualitative reverse parking driver characteristics. Based on the determined characteristics, we propose a haptic assistance calculation method that utilizes the receding horizon control algorithm. For a simulation environment to assess the proposed assistance method, we also developed a scaled car simulator comprising a $1 / 10$ scaled robot car and an omnidirectional camera. We used the scaled car simulator to conduct comparative experiments on subjects, and observed that the driving skills of the assisted subjects were significantly better than those of the control subjects.
\end{abstract}

Index Terms—Haptic assistance, Driving skills, Human behavior analysis, Optimal control.

\section{INTRODUCTION}

A SSISTED driving technologies on a haptic assistance have become popular in recent years. Compared to conventional assisted driving systems based on visual and/or auditory assistance [1][2][3], the use of haptic modality is expected to afford more effective and less disturbing assistance to drivers. Griffiths et al. examined the potential of haptic assistance performing driving tasks in a virtual environment [4]. It was observed that haptic assistance improved the performance of the subjects in the pathfollowing, and obstacle-avoidance tasks, and also reduced the visual demand during the tasks. Moreover, as Navarro et al. noted, several assisted driving systems that use haptic interventions for assisting lanekeeping and curve-negotiation have been proposed [5]. For example, Saleh et al. proposed a control sys-

- M. Hirokawa is with Graduate School of Systems and Information Engineering, University of Tsukuba, Tsukuba, Ibaraki, 305-8573 Japan. Research Fellow of the Japan Society for the Promotion. e-mail: hirokawa@ai.iit.tsukuba.ac.jp

- N. Uesugi and T. Kitagawa are with Mazda Motor Corporation, Technical Research Center, Advanced Vehicle System Research Field, Yokohama, Kanagawa, Japan. email: uesugi.n, kitagawa.t@mazda.co.jp

- S. Furugori is with Mazda Motor Corporation, Technical Research Center, Innovative Research Planning Field, 2-5 Moriyacho Kanagawa-ku, Yokohama, Kanagawa, Japan. email: furugori.s@mazda.co.jp

- K. Suzuki is with Faculty of Engineering, Information and Systems, University of Tsukuba and with Japan Science and Technology Agency, 1-1-1 Tennoudai, Tsukuba, Ibaraki, Japan. e-mail: kenji@ieee.org tem shared by the human driver and the haptic assistance system, which takes into account the uncertainty in the behavior of the driver by means of a cybernetic driver model for lane-keeping [6][7]. Furthermore, Deroo et al. demonstrated a lane-departure warning system that improves the steering response of the driver by haptic stimulation without eliciting a compensatory reflex reaction[8]. Mulder et al. proposed an assisted driving system that uses haptic feedback to guide the driver when negotiating a curve [9]. Simulation experiments revealed that drivers assisted by the haptic guidance system exhibited better driving behavior and negotiated the curve more safely. To assist pedal control, Kuge et. al. and Azzi et. al. developed a haptic assistance system that uses force feedback on the gas pedals [10][11].

However, although driving with haptic assistance can be considered as a human-machine cooperative task [12], haptic assistance systems have basically so far been evaluated from the viewpoints of the safety and stability of using them, and their effect on human driving behavior has not been sufficiently discussed. Therefore, assessment of the effect of the haptic intervention of the system on the skill of the driver would also be beneficial for improving the model of human driving behavior by the inclusion of a mechanism for adapting human interaction with the assistance system.

In this paper, we introduce an assisted driving method based on haptic assistance for training drivers in reverse parking, which is considered an uncertain factor in conventional assisted driving systems. In the 
attempt to develop such a training system, we have first focused on reverse parking. Because reverse parking is relatively difficult (i.e., requiring greater skill) than other driving processes, and has also not been previously studied, it is appropriate for developing an assistance system for training drivers. The proposed system assists a driver by applying a guidance torque on the steering wheel during reverse parking, to encourage the driver to operate the steering wheel in a proper way and with correct timing. When using this system, the driver can intuitively recognize the guidance intention in the same manner as a trainer teaches steering operation from the navigation seat at a driving school.

Apart from the proposed approach, there is another approach to delegating the entire operation of reverse parking to the system. Automatic reverse parking has been well studied, and several systems are presently commercially available. The Intelligent Parking Assist System, which is capable of automatic reverse parking was developed by Toyota Motor Corporation and is in practical use [13]. Moreover, Honda Motor Corporation has developed a parking assistance system from the perspective of motion assistance [14]. Basically, in conventional mobility assistance systems, all the operations necessary for accomplishing reverse parking are automatically controlled by the system. Consequently, beginner drivers cannot improve their driving skills by using such systems. Moreover, some negative consequences of automation such as the outof-the-loop performance problem, loss of situational awareness, complacency (over-trust), and automation surprises, have been pointed out [15][16]. The locus of responsibility when an accident occurs while using an automated system is another issue in need of discussion. Further, a fully autonomous vehicle requires more time for the practical realization and for the setup of appropriate infrastructures [17]. Even when fully automated vehicles become widely available, the conventional interface for manual control will not be removed because of security concerns. For these reasons, it is always better to improve the skill of drivers, regardless of the car being used.

This paper is organized as follows. In the next section, we propose our haptic assistance method for driving training, and describe a simulation environment that we developed for its experimental implementation and validation. An experiment conducted to determine human driving characteristics using a real car is described in Section III. Based on the characteristics of successful driving, we developed an assistance algorithm, which is described in Section IV. In Section V, we describe an experiment in which the simulator was used to validate the effectiveness of the proposed method for improving the reverse parking skill of a driver. Finally, in Section VI, we present a summary and draw some conclusions.

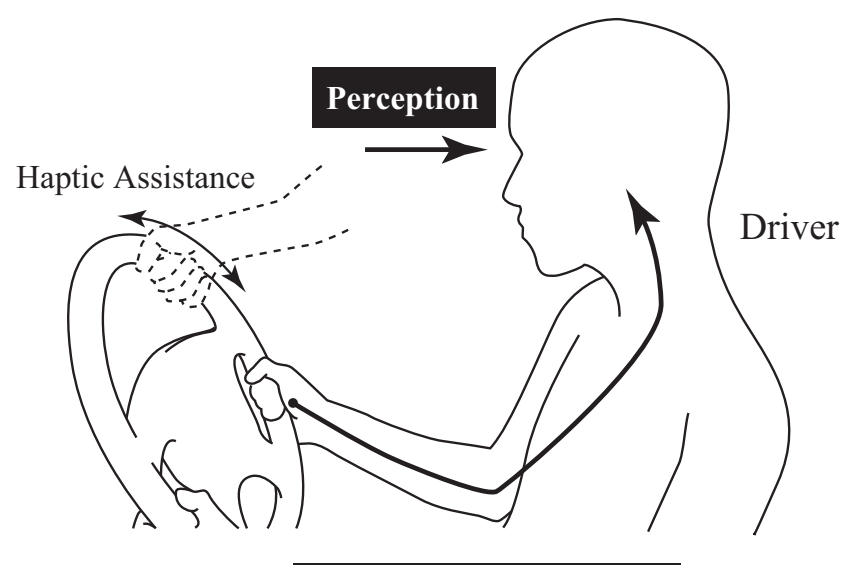

Somatosensory Feedback

Fig. 1. The proposed approach for steering operation training. A guidance torque is applied on the motorized steering wheel to induce the driver's operation by somatosensory feedback. The guidance torque is calculated by a real-time optimal control method known as receding horizon with regard to the current vehicle situation.

\section{METHODOLOGY}

\subsection{Haptic Assistance for Driving Training}

Haptic assistance has been known as an efficient approach for training motor control skill of human, especially rehabilitative training on motor recovery. MIT-Manus was developed to help impaired people to relearn their motor function of arm by haptic assistance [18]. Vehicle driving, especially handling of the steering wheel, is a kind of motor control task based on cognition; therefore, real-time haptic assistance for handling the steering wheel can be used to facilitate human cognitive-motor skills (in this case, driving skills). Yang et al. have developed a visuohaptic(visual + haptic) system for path-following training [19]. In addition, Powell et al. conducted a comparative study of haptic guidance technologies applied to two different dynamic tasks[20]. In this work, they showed that haptic-based guidance improves the motor control skill of the user by reducing the required effort.

Figure 1 illustrates our approach for steering operation training by haptic intervention. A guidance torque is applied on the motorized steering wheel to induce the intentional operation by the driver through somatosensory feedback during reverse parking. As described by Asseldonk et al., the use of haptic intervention for motor guidance can be categorized into several groups such as error enhanced, soft guidance, hard guidance and passive [21]. According to Asseldonk, error enhanced (which amplifies an operation error of the driver) and hard guidance (which constrains the movement range) are inappropriate for actual driving situations. Moreover, for successful reverse parking, drivers need to learn appropriate 


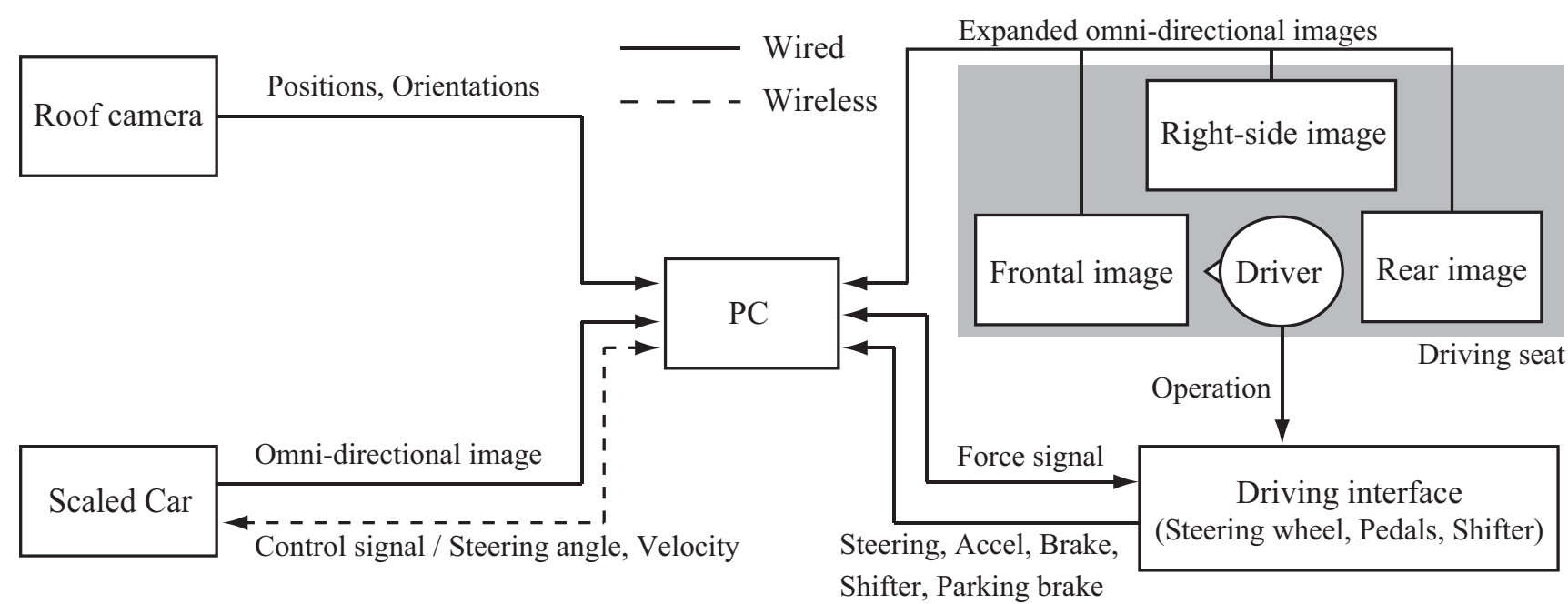

Fig. 2. Overview of the scaled car simulator system. It consists of a $1 / 10$ scaled remotely controllable car, an omnidirectional camera, and a separate driving environment that includes a driving seat, a steering wheel, and accelerator and brake pedals.

timing for rotating the steering wheel; thus, passive guidance, which does not involve active steering wheel control, is not effective for driving training. For these reasons, we adopted soft guidance, in which a guidance force is generated to compensate for the error in the desired angle of the steering wheel,. Its strength is proportional to the error. Moreover, in the study of Asseldonk, the participants exhibited strong after-effects of the soft guidance condition. In other words, the cognitive-motor behavior learned by soft guidance was maintained longer after the assistance was removed than those of the other conditions. In this sense, soft guidance is more appropriate for the purpose of explicitly affecting the human learning process and improving safety.

As a related work with similar objective, the Intelligent Drive Master was produced by Mazda Motor Corporation to support the process of learning how to drive [22]. The system aimed at improving the fuel consumption and driving comfort by training in driving techniques. The system models the movement of the vehicle and the center of gravity of the driver based on sensory data obtained during driving. The model categorizes the driving behavior as soft, harsh or pliant, and feeds it back to the driver as simple visual information. However, the visual feedback is not sufficient for determining how to refine the driving behavior of the driver because the driving operation is composed of several simultaneous actions involving various interfaces. From this perspective, our proposed method is advantageous because it supplies the desired operation in an intuitive manner.

\subsection{Scaled Car Simulator}

We developed the $1 / 10$ scaled car simulator shown in Fig. 3 as an experimental environment for implement-

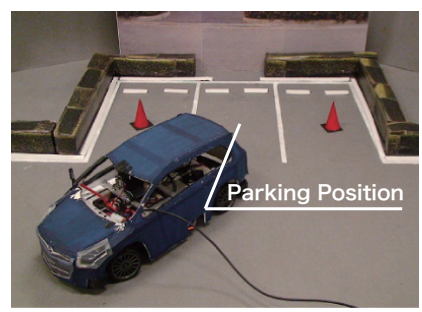

(a)

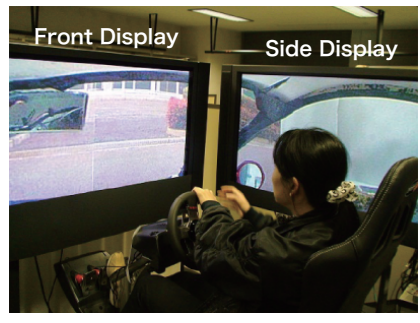

(c)

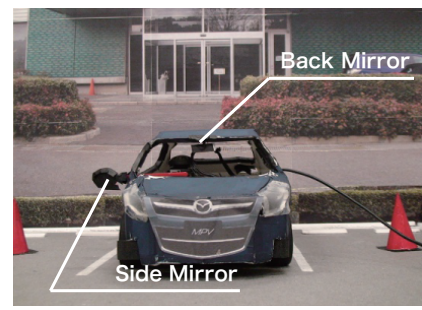

(b)

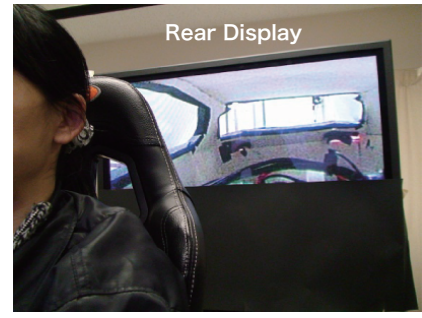

(d)
Fig. 3. Scaled car simulator consisting of a $1 / 10$ scaled remotely controllable car, an omnidirectional camera, and a separate seat with a driving controller. (a) Bird's eye view, (b) Front view, (c) Front view from the driving seat, and (d) Rear view from the driving seat.

ing and verifying the proposed assistance system. The simulator consists of a $1 / 10$ scaled remotely controllable car (ZMP Corporation), an omnidirectional camera which is mounted in the driver's position in the scaled car, and a separate driving environment that includes a driving controller with a steering wheel and acceleration and brake pedals. The subject was requested to sit on the stationery driving seat and remotely control the scaled car. An actuator was installed on the rotational axis of the steering wheel and the haptic assistance could be fed to the 
TABLE 1

Measurement parameters

\begin{tabular}{|c|c|c|c|c|}
\hline Measured parameters & Measuring method & Range & Resolution & Sampling rate \\
\hline \hline Steering angle & CAN & $-200 \sim 200\left[{ }^{\circ}\right]$ & $1\left[^{\circ}\right]$ & $10[\mathrm{~Hz}]$ \\
Brake pedal pressure & CAN & $-2.45 \sim 22.102[\mathrm{MPa}]$ & $0.024[\mathrm{MPa}]$ & $10[\mathrm{~Hz}]$ \\
Accelerator pedal pressure & CAN & $0 \sim 100[\%]$ & $0.5[\%]$ & $10[\mathrm{~Hz}]$ \\
Velocity & CAN & $0 \sim 300[\mathrm{Km} / \mathrm{h}]$ & $0.01[\mathrm{Km} / \mathrm{h}]$ & $10[\mathrm{~Hz}]$ \\
Velocity of wheels & CAN & $0 \sim 300[\mathrm{Km} / \mathrm{h}]$ & $0.01[\mathrm{Km} / \mathrm{h}]$ & $10[\mathrm{~Hz}]$ \\
Parking brake & CAN & - & ON/OFF & $10[\mathrm{~Hz}]$ \\
Shift lever & CAN & - & 6 states & $10[\mathrm{~Hz}]$ \\
Position & GPS(RTK) & Entire experiment area & & $10[\mathrm{~Hz}]$ \\
\hline
\end{tabular}

driver through the wheel by driving the actuator. The steering wheel was of diameter $28[\mathrm{~cm}]$ and had a rotation range of $\pm 450\left[^{\circ}\right]$. Exterior and interior equipment, for example the rear view mirror and side mirror, were developed. The omnidirectional image was expanded into normal images and displayed on monitors surrounding the driving seat of the simulator as shown in Fig. 3. Thus, the subject could see the views from the driving seat of the scaled car as if they were sitting in the front seat of the scaled car. The real image display afforded the driver a realistic impression, and the simulator could create various driving situations by placing scaled objects around the scaled car, for example to imitate buildings and obstacles. The central computer controlled the scaled car via wireless connection based on the operation input of the driver. The control cycle was set to $100 \mathrm{~ms}$ owing to the limitation of the communication speed. Information about the position and orientation of the scaled car and the target parking space were obtained by detecting AR markers attached to both the car and the parking space by means of a camera hanged above the experimental environment.

\section{QUALITATIVE CHARACTERIZATION OF HUMANS DRIVING}

\subsection{Experimental Procedure}

To design the appropriate haptic assistance method for reverse parking steering operation training, we conducted a measurement experiment to determine the qualitative characteristics of humans driving behavior in real situations. In the experiment, the subjects were required to perform reverse parking 10 times from two initial positions (right and left, alternately) to the parking space indicated by crosshatching as shown in Fig. 4. During each trial, the subjects were allowed to change direction (i.e., change the moving direction from forward/backward to backward/forward) only once. All the 10 subjects (nine males and one female) who participated in the study held regular driving licenses (4 30 years) and gave their informed consent. The measured parameters shown in Table I were recorded by measuring instruments mounted on the experimental vehicle, which was a station wagon. To evaluate the parking scores,

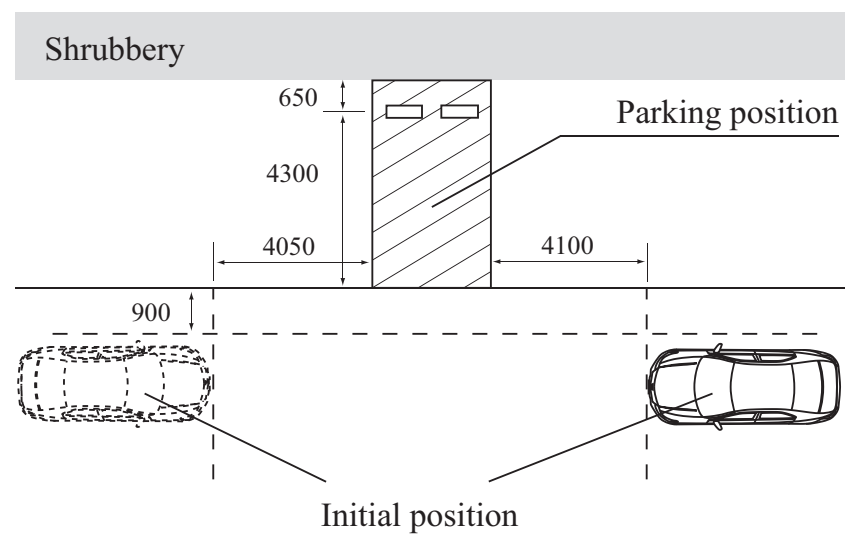

Unit: [mm]

Building

Fig. 4. Overview of an experimental measurement environment. The subjects were required to perform reverse parking from two initial positions to the target parking position.

TABLE 2

Criteria for scoring the parking.

\begin{tabular}{|c|c|c|}
\hline Score & Position & Orientation \\
\hline \hline 4 & Center of parking area & Straight \\
3 & Inside lines but lopsided & Slightly tilted \\
2 & One or more wheels on the line & Tilted \\
1 & One or more wheels beyond the line & Very tilted \\
\hline
\end{tabular}

the top view of the experimental field was recorded by a video camera.

\subsection{Quantification of Parking Scores}

To analyze the relationship between the driving actions during parking and the resulting performance, we first quantified the parking score of each subject according to the parking position, orientation, and the time taken to complete the parking. The parking position and orientation were evaluated on a fourdegree Likert scale using a human coder based on the top view videos recorded during the experiment. The criteria for the evaluation are described in Table II. Each trial was independently evaluated by three coders, and the averaged rating was used for 


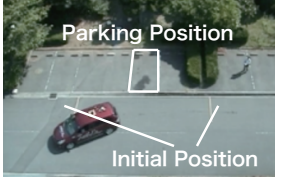

(a) Feild overview

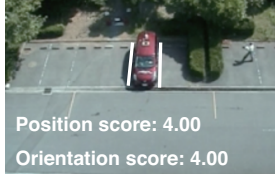

(b) Successful parking

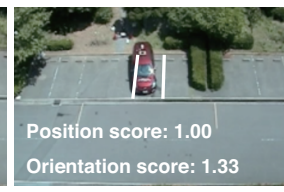

(c) Failed parking
Fig. 5. Examples of successful (middle) and failed (right) parking, and averaged scores of the subjects.

TABLE 3

$\kappa$ coefficients among human coders (Position/Orientation)

\begin{tabular}{|c||c|c|c|}
\hline & Experimenter A & Experimenter B & Experimenter C \\
\hline \hline A & 1 & $0.935 / 0.925$ & $0.889 / 0.917$ \\
\hline B & $0.935 / 0.925$ & 1 & $0.926 / 0.953$ \\
\hline C & $0.889 / 0.917$ & $0.926 / 0.953$ & 1 \\
\hline
\end{tabular}

the analysis. Figure 5 shows examples of successful (middle) and failed (right) parking, and the averaged position/orientation scores.

To verify the human evaluation, we examined the similarities among the results of the coders. Table III shows the Kappa correlation coefficients among the coders; the minimum value for all the combinations is 0.889 . This means that the evaluations of the three human coders were sufficiently coherent to be reliably used for analysis. In addition to the parking position and orientation, the elapsed time between the direction-change-point, when the car started going backward, and the completion of parking was also measured. The parking score, which indicates how precisely and quickly the subject completed the parking, was defined as the sum of the parking position/orientation score and the required time score. It should be noted that, to combine both scores, they were normalized to the same offset and standard deviation using the following equation:

$$
x_{i}^{\prime}=\frac{x_{i}-\mu}{\sigma}+x_{o f f s e t}
$$

where $\mu, \sigma$ respectively denote the average and standard deviation, and $x_{\text {of } f \text { set }}$ is set to 3 for the following analysis. The following analysis was conducted done for all the subjects except one whose video data could not be recorded.

\subsection{Analysis}

To investigate the characteristics of human driving and their relationship with the parking score, the operation histories of the steering and the acceleration and brake pedals after the direction-change were analyzed. When a series of operation data $\{x(t) \mid 1 \leq$ $t \leq T\}$ is obtained via the internal CAN network of the car, the amount of the driver's operation can be

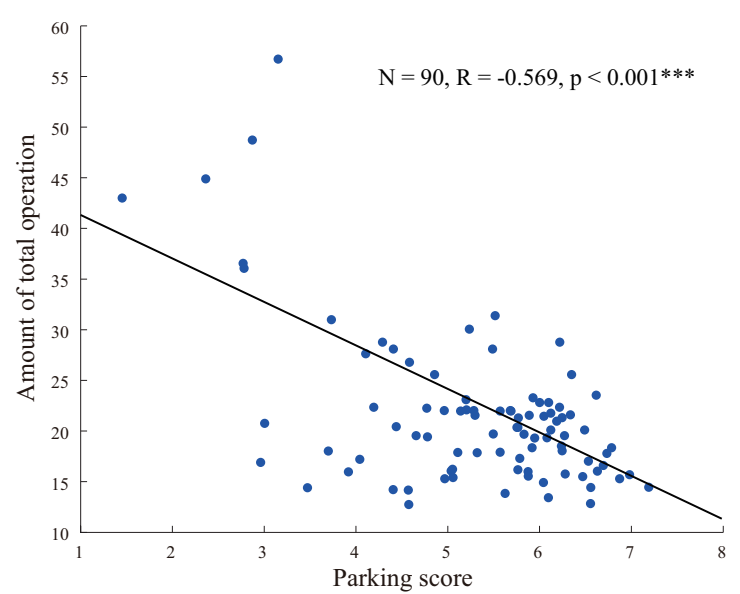

Fig. 6. Results of analyzing the correlation between the parking score and the total amount of operation. A negative correlation can be observed ( $p \leq 0.001$, ttest).

quantified using the following equation:

$$
\begin{aligned}
\Delta x(t) & =|x(t)-x(t-1)| \\
M & =\frac{1}{\max x} \sum_{t=1}^{T} \Delta x(t)
\end{aligned}
$$

The total amount of operation $M_{\text {total }}$ can be calculated as the sum of the amount of steering wheel operation $M_{s t r}$, the acceleration pedal operation $M_{a c c}$ and the brake pedal operation $M_{b r k}$ as follows:

$$
M_{\text {total }}=M_{\text {str }}+M_{a c c}+M_{b r k}
$$

Figure 6 shows the correlation between the total amount of operation and the parking score. The results indicate a negative correlation with a correlation coefficient of -0.569 and a $p \leq 0.001$ significance level for a double-ended t-test.

Next, we investigated the relationship between the steering of the driver while changing the velocity of the vehicle and the parking score. The operation of the steering wheel while changing the velocity of the vehicle $\Delta x_{s t r}^{\prime}$ and the change in the vehicle velocity $\Delta x_{v}^{\prime}$ were determined using equations 5 and 6 :

$$
\begin{aligned}
\Delta x_{s t r}^{\prime}(t) & =\left|x_{s t r}(t)-x_{s t r}(t-1)\right|\left[\Delta x_{v} \neq 0\right] \\
\Delta x_{v}^{\prime}(t) & =\left|x_{v}(t)-x_{v}(t-1)\right|\left[\Delta x_{s t r} \neq 0\right]
\end{aligned}
$$

Then, the total amount of operation with velocity change $M_{v c}$ can be defined as follows:

$$
\begin{aligned}
M_{s t r}^{\prime} & =\frac{1}{\max x_{s t r}} \sum_{t=2}^{T} \Delta x_{s t r}^{\prime}(t) \\
M_{v}^{\prime} & =\frac{1}{\max x_{v}} \sum_{t=2}^{T} \Delta x_{v}^{\prime}(t) \\
M_{v c} & =M_{s t r}^{\prime}+M_{v}^{\prime}
\end{aligned}
$$




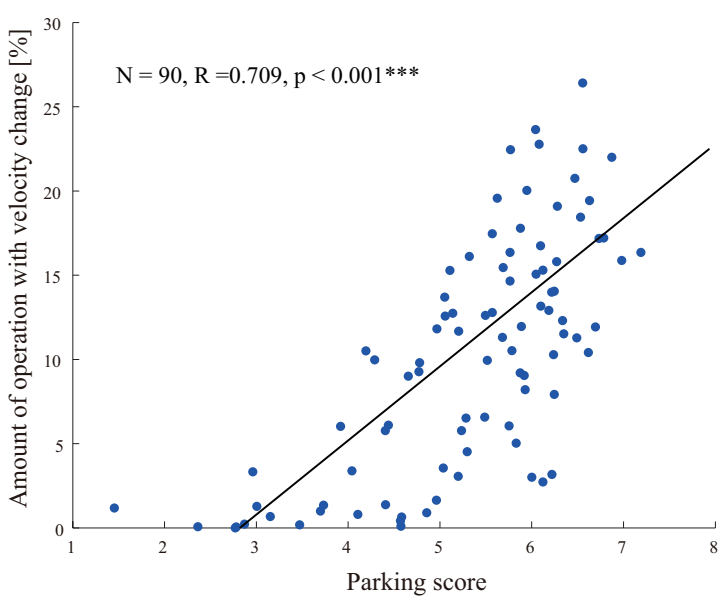

Fig. 7. Results of analyzing the correlation between the parking score and the amount of operation with velocity change. A positive correlation can be observed $(p \leq 0.001$, t-test).

Afterwards, the ratio of the amount of operation with velocity change $M_{v c}$ to the total amount of operation $M_{\text {total }}$ was obtained using Eq. 10.

$$
R_{v c}=M_{v c} / M_{t o t a l}
$$

Figure 7 illustrates the relationship between the amount of operation with velocity change and the parking score. The results of the correlation analysis indicate that there is a significant positive correlation with a $p \leq 0.001$ significance level for a double-ended t-test.

It should be noted that the average parking score of subjects using a same type car as the experimental vehicle in their daily life were not significantly different from that of other subjects. Therefore, the effect of the type of the experimental vehicle to the parking score was negligible.

\subsection{Discussion}

From the experimental results, we obtained several insights into the characteristics of human driving. Regarding the relationship between the total amount of operation and the parking score for the trials shown in Fig. 6, a significant negative correlation was observed. This means that a driver with a higher parking score completed the parking quickly and accurately with a lower amount of operation than a driver with a lower parking score. This is in agreement with our expectations regarding the driving skill and the total amount of operation; i.e., the operations of an experienced driver are refined to reduce redundancies such as modifications and corrections.

Furthermore, Fig. 7. reveals a significant positive correlation between the amount of operation with velocity change and the parking score. This means that operation of the steering in accordance with the velocity change increases the speed and precision of the parking trial. However, for trials with lower scores, the drivers operated the steering wheel while driving the car at a constant speed, or while the car was stationary, which is the so-called stationary steering. A possible explanation for this trend is that steering with velocity change requires higher cognitive skills to estimate the prospective movement of the car. This difficulty leads to the incorrect operation by inexperienced drivers, and the characteristics have been identified by by other studies on experienced driving [23].

\section{Haptic Assistance}

Based on these findings about the characteristics of the operation behavior of drivers during successful parking, we defined a target operation behavior for the proposed assistance system to minimize the total amount of operation and the amount of operation with velocity change. We then developed an algorithm that assists reverse parking by automatically minimizing the total amount of operation quantity and the time taken through control of the steering wheel. It consists of (i) a path-planning algorithm for deriving the optimal path from the point of direction change to the parking position, and (ii) an optimal control-based algorithm for controlling the steering wheel to follow the path. It should be noted that, because the position of the point of direction change is related to the difficulty of parking, automated parking systems require the driver to initiate the system from a predetermined kinematically easy direction-change point regardless of the prevailing situation [13]. Thus, instead of developing a system that determines the optimal point of direction-change by recognizing the environment, we developed one that allows the driver to choose the point of direction change and assists the parking from the chosen point, unless it is kinematically inappropriate.

\subsection{Path Planning}

Figure 8 illustrates the path-planning algorithm from the direction-change point based on the Bezier curve. The clothoid curve, which is a kind of relaxation curve with increasing curvature has been used in some practical parking assistance systems as a path-planning algorithm for controlling four-wheeled vehicles. The points $(x, y)$ on the clothoid curve are expressed in integral form as follows:

$$
x(l)=\frac{1}{a} \int_{0}^{l} \cos s^{2} d s, \quad y(l)=\frac{1}{a} \int_{0}^{l} \sin s^{2} d s
$$

Therefore, when the initial and destination points (in this case, the direction-change point and the parking position) are given, it is difficult to use an analytical approach to solve the inverse problem in deriving 


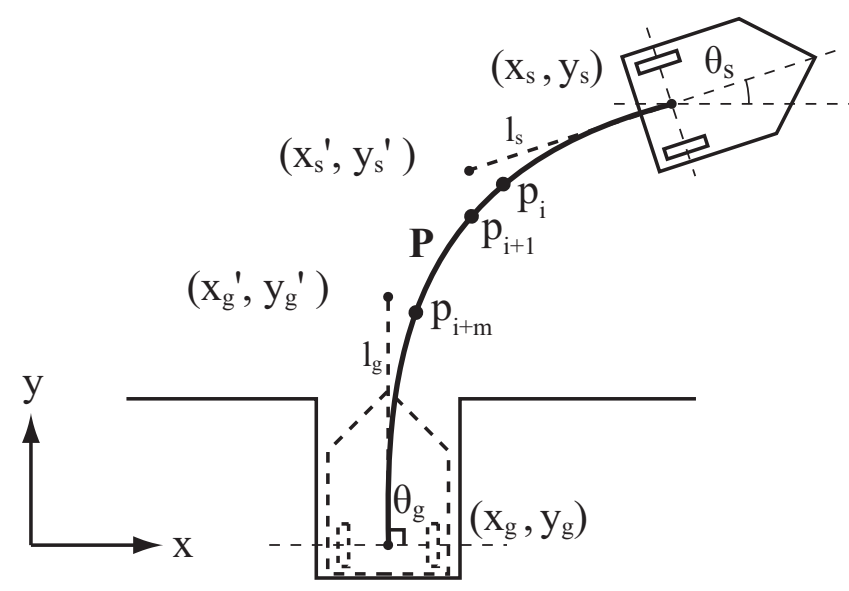

Fig. 8. Path-planning algorithm based on the Bezier curve.

the appropriate curvature parameter $a$ because an iterative calculation that is not suitable for real-time assistance is required.

However, the Bezier curve can be quickly created between any two points by manipulating the curvature of each terminal point. It is also possible to approximate the actual vehicle trajectory to the clothoid curve by regulating the maximum angular velocity of the steering wheel during the process of calculating the optimal input.

$\left(x_{s}, y_{s}, \theta_{s}\right)$ denotes the initial position and the orientation of the vehicle at the direction-change point, respectively, and $\left(x_{g}, y_{g}, \theta_{g}\right)$ denotes the target position in the parking space. $l_{s}$ and $l_{g}$ are the lengths of the tangential lines associated with the curvature at the terminal points. Thus, based on $x_{s}, y_{s}, \theta_{s}, l_{s}, l_{g}$, the control points $\left(x_{s}^{\prime}, y_{s}^{\prime}\right),\left(x_{g}^{\prime}, y_{g}^{\prime}\right)$ can be determined as follows:

$$
\begin{aligned}
& \left(x_{s}^{\prime}, y_{s}^{\prime}\right)=\left(l_{s} \cos \left(\theta_{s}+\pi\right), l_{s} \sin \left(\theta_{s}+\pi\right)\right) \\
& \left(x_{g}^{\prime}, y_{g}^{\prime}\right)=\left(l_{g} \cos \theta_{g}, l_{g} \sin \theta_{g}\right)
\end{aligned}
$$

If $n$ denotes the number of points that form the Bezier curve, the curve $P=\left\{\boldsymbol{p}_{i}\left(x_{i}, y_{i}\right) \mid i=0 \cdots n\right\}$ can be obtained using the following equations:

$$
\begin{gathered}
t=i / n \quad(0 \leq t \leq 1) \quad \tau=(1-t) \\
x_{i}=\tau^{3} x_{s}+3 t \tau^{2} x_{s}^{\prime}+3 t^{2} \tau x_{g}^{\prime}+t^{3} x_{g} \\
y_{i}=\tau^{3} y_{s}+3 t \tau^{2} y_{s}^{\prime}+3 t^{2} \tau y_{g}^{\prime}+t^{3} y_{g} \\
\theta_{i}=\arctan \left(\frac{y_{i}-y_{i+1}}{x_{i}-x_{i+1}}\right)
\end{gathered}
$$

The length of the curve $L$ and the maximum curvature $\kappa$ are obtained as follows:

$$
\begin{aligned}
& L=\sum_{i=0}^{n} \sqrt{\left(x_{i}-x_{i+1}\right)^{2}+\left(y_{i}-y_{i+1}\right)^{2}} \\
& \kappa=\max _{i}\left|\frac{\dot{x}_{i} \ddot{y}_{i}-\dot{y}_{i} \ddot{x}_{i}}{\left(\dot{x}_{i}{ }^{2}+\dot{y}_{i}{ }^{2}\right)^{\frac{3}{2}}}\right|
\end{aligned}
$$

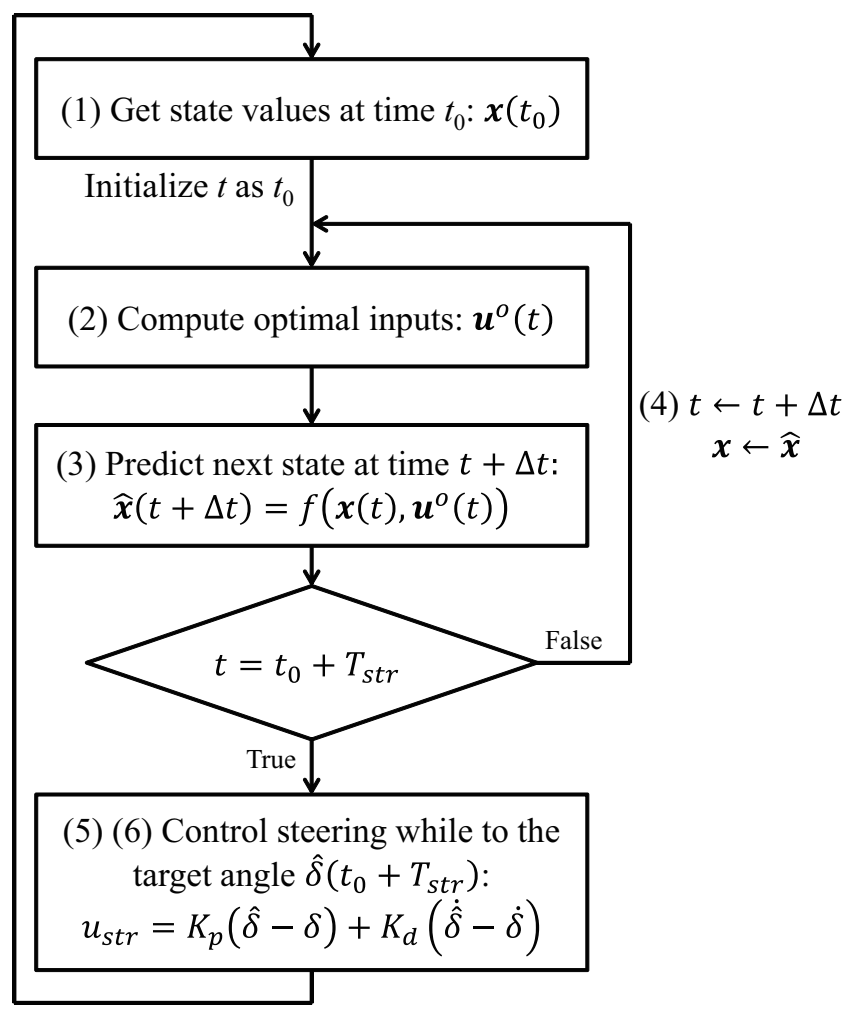

(7)

Fig. 9. Flow chart of calculation of haptic assistance.

The optimal path $P_{o p t}$ is determined as the shortest path among those with maximum radii of curvature $(1 / \kappa)$ larger than the minimum turning radius of the vehicle. The algorithm explores the optimal path by changing $l_{s}, l_{g}$ within the limited range.

Owing to the kinematic constraints of the vehicle and the number of changing directions, all reverse parking cannot be accomplished from all the direction-change points. In the validation experiment described in Section V, if the system recognizes that it is physically impossible to calculate the appropriate path from the current direction-change point, the subject would be notified via LEDs mounted on the steering wheel, and would then continue reverse parking without the support of the system.

\subsection{Calculation of Haptic Assistance}

To use haptic intervention for real-time operation assistance, the time delay in controlling the actuator should be considered. Therefore, a steering control algorithm, that is capable of generating haptic assistance with appropriate timing while taking the time constant of the actuator into consideration is required. It is difficult to handle this using a typical feedback controlling framework that decides the input signal for the next time step based on the error between the current steering angle and the target angle. To solve this problem, the target angle in controlling 
the steering wheel should be derived from the future steering angle predicted on the basis of the present vehicle state. For this reason, we adopted the receding horizon control method to determine the haptic assistance [24][25]. In the receding horizon method, the input values are calculated by predicting the shortterm future states at every time step, whereas in regular optimal control methods, the input values are calculated in advance. Receding horizon is therefore more robust against noise and more appropriate for real-time control in terms of reducing the calculation cost.

The haptic assistance is computed based on the predicted state values of the vehicle, which are obtained during the calculation process of the receding horizon. The state values of the vehicle $\boldsymbol{x}=\{x, y, \theta, \delta\}$, the input values $\boldsymbol{u}=\left\{u_{1}, u_{2}\right\}$, and the state equation are determined as follows:

$$
\dot{\boldsymbol{x}}=f(\boldsymbol{x}, \boldsymbol{u}, t)=\left[\begin{array}{c}
u_{1} \cos \theta \\
u_{1} \sin \theta \\
u_{1} \frac{\tan \delta}{D} \\
u_{2}
\end{array}\right]
$$

$(x, y)$ denotes the position, $\theta$ denotes the orientation and $\delta$ denotes the steering angle. $u_{1}$ is the control input for the velocity, and $u_{2}$ is the control input for the angular the velocity of steering. $D$ denotes the wheel base width of the vehicle.

Figure 9 shows the flow chart of the calculation of the haptic assistance. The execution steps are as follows:

1) Determine the point $\boldsymbol{p}_{i}$ on the derived path $P_{\text {opt }}$, that is nearest to the position of the current vehicle $\boldsymbol{x}\left(t_{0}\right)$ at time $t_{0}$.

2) Define the point $\boldsymbol{p}_{j}$ as an temporal goal, where $j=i+m$ and $m$ is a constant positive integer, and solve the optimal control problem for the interval $t_{0} \leq t \leq t_{0}+T_{f}$. The series of optimal inputs $U^{o}=\left\{\boldsymbol{u}^{o}(t) \mid t_{0} \leq t \leq t_{0}+T_{f}\right\}$ is then obtained. The evaluation function that is used for the calculation is

$$
\begin{aligned}
J & =\int_{0}^{T_{f}}\left(\boldsymbol{x}_{g}-\boldsymbol{x}(t)\right)^{T} Q\left(\boldsymbol{x}_{g}-\boldsymbol{x}(t)\right) d t \\
& +\int_{0}^{T_{f}} \boldsymbol{u}(t)^{T} R \boldsymbol{u}(t) d t \\
& +\left(\boldsymbol{x}_{g}-\boldsymbol{x}\left(T_{f}\right)\right)^{T} S\left(\boldsymbol{x}_{g}-\boldsymbol{x}\left(T_{f}\right)\right)
\end{aligned}
$$

$\boldsymbol{x}_{g}$ denotes the target position and orientation, and $Q, R$, and $S$ are the weight matrices. The first and last members of the evaluation function are regarded as the minimization of errors in the position/orientation of the vehicle, and the middle member represents the accumulated amount of operation. Using this evaluation function, an optimal input $U^{o}$ that minimizes both the control error and the total amount of operation is therefore obtained. Moreover, $U^{o}$ is set to zero when the vehicle is not moving in order to inhibit the stationary steering and increase the amount of operation with velocity change.

3) Predict the future state values of the vehicle $\hat{\boldsymbol{x}}\left(t_{0}+\Delta t\right)$ using $\boldsymbol{u}^{o}\left(t_{0}\right)$, the optimal input at time $t_{0}$ and the state equation $f$.

4) Iterate the above steps by reassigning $\boldsymbol{x} \leftarrow \hat{\boldsymbol{x}}$ and $t_{0} \leftarrow t_{0}+\Delta t$ to generate the series of predicted state values $\hat{X}=\left\{\hat{\boldsymbol{x}}(t) \mid t_{0} \leq t \leq t_{0}+T_{\text {str }}\right\}$, where $T_{\text {str }}$ denotes the time constant of the step response of the steering wheel.

5) Determine the estimated angle of the steering wheel $\hat{\delta}\left(t_{0}+T_{s t r}\right)$ as the target angle for controlling the steering wheel.

6) Calculate an input for controlling the steering wheel $u_{\text {str }}$ using the following PD control equation:

$$
u_{s t r}=K_{p}(\hat{\delta}-\delta)+K_{d}(\dot{\hat{\delta}}-\dot{\delta})
$$

$K_{p}$ and $K_{d}$ respectively represent the proportional and differential gains, and $\dot{\hat{\delta}}$ is the target angular velocity, which is always zero. The applied force is chosen to be between 0.2 [N] upto $0.6[\mathrm{~N}]$, and is proportional to the error in the desired steering angle. The control of the steering wheel runs at $1[\mathrm{kHz}]$ as a thread independent of the haptic assistance calculation process.

7) Repeat the above process using a $10[\mathrm{~Hz}]$ control cycle.

The set of control gains $K_{p}$ and $K_{d}$ was empirically determined so that all the users could physically rotate the steering wheel in the direction opposite to that intended by the system. For the following experiment, we set the stiffness parameter $K_{p}$ to 200, and the damping parameter $K_{d}$ to 10 . We confirmed that fully automated reverse parking could be accomplished using these parameters when no load is applied to the steering wheel; i.e., the driver does not hold the wheel. The determined parameters were uniformly applied for all the subjects.

\section{EXPERIMENT}

\subsection{Experimental Procedure}

To validate the proposed method, we conducted an experiment in the scaled car simulator environment. The experimental task was reverse parking and 10 subjects (eight males and two females, with $12-30$ years driving experience) participated in the experiment. Eight of the 10 subjects also participated in the experiment described in Section III. The effect of the previous experiment was considered to be negligibly small because the experiment was conducted after several months. The subjects were required to perform reverse parking into a slot between two red cones, which could be visualized through the windows and mirrors of the simulator. The experiment 
TABLE 4

Assistance conditions

\begin{tabular}{|c|c|c|c|}
\hline & $\begin{array}{c}1^{\text {st }} \text { set } \\
(1 \sim 5 \text { trials })\end{array}$ & $\begin{array}{c}2^{\text {nd }} \text { set } \\
(6 \sim 10 \text { trials })\end{array}$ & $\begin{array}{c}3^{\text {rd }} \text { set } \\
(11 \sim 15 \text { trials })\end{array}$ \\
\hline \hline Group A & non-assisted & non-assisted & non-assisted \\
\hline Group B & non-assisted & assisted & non-assisted \\
\hline
\end{tabular}

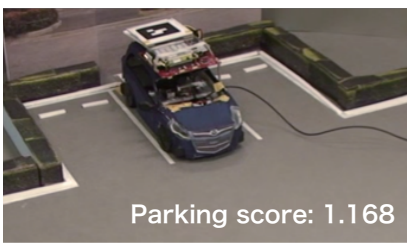

(a) Successful parking

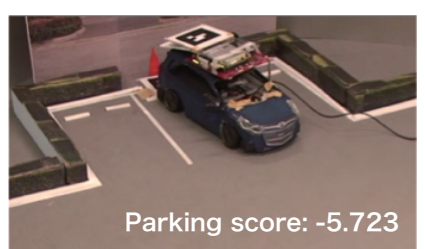

(b) Failed parking
Fig. 10. Examples of (a) successful and (b) failed parking in the simulation experiment, and the normalized parking scores.

field in which the scaled car moved was surrounded by monocolored partitions to hide other objects in the room. The subjects were divided into groups A and $B$ for the experiment. One group was the target group of assisted drivers, and the other one was the control group, which was used for comparative study. Each subject was allowed 15 parking trials, which were divided into three sets (five trials per set). As in the measurement experiment in Section III, each subject was allowed to change the direction of the car only once. The state value of the vehicle and the operation information for each subject were recorded for further analyses. It should be noted that two participants were excluded from the analysis. One was a subject who participated in the preliminary experiment used for establishing the experimental procedures, and the other was one who could perform park very well before the experiment (the parking score of this subject was significantly better than those of the other subjects, with more than $99 \%$ significance for a double-ended $t$-test).

The conditions of the experiment are given in Table 4 . The proposed assistance was only applied in the $2^{\text {nd }}$ set of Group B to verify the effect of the assistance by comparing the average progress of the different groups. In the $2^{\text {nd }}$ set of Group B, the assistive function was activated when the vehicle reached the direction-change point and the driver shifted the gear into "reverse". It should be noted that, if the path planning failed as a result of an inappropriate direction-change point, the system would inform the driver by blinks in the LEDs mounted on the steering wheel, and the haptic assistance would not work in the parking trial.

\subsection{Analysis of Parking Score}

To analyze the results of the experiment, we first quantify the parking score in the same manner as

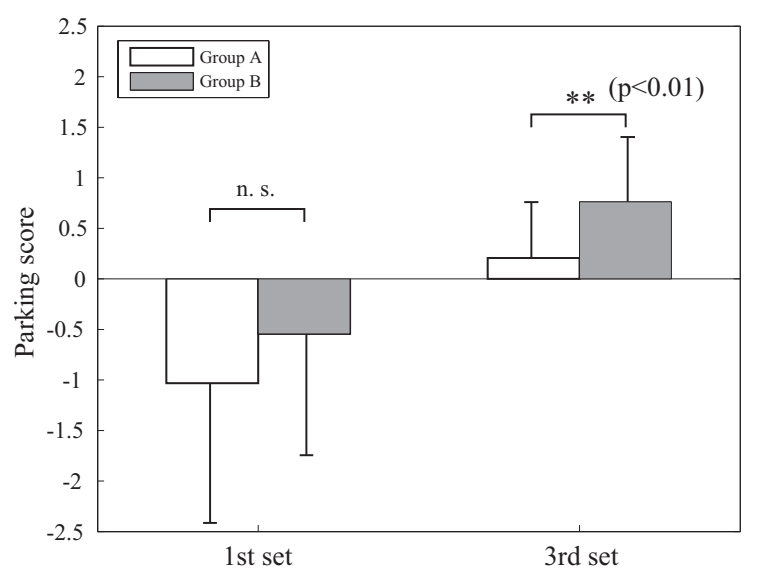

Fig. 11. Comparison of averaged parking scores of the groups. There is significant difference for a MannWhitney's U-test $(p<0.01)$ in the $3^{\text {rd }}$ set, whereas no difference was observed in the 1 st set.

TABLE 5

Relationship between the direction-change point (DCP) and the rate of successful parking

\begin{tabular}{|c|c|c|c|c|}
\hline & \multicolumn{2}{|c|}{ Group A } & \multicolumn{2}{|c|}{ Group B } \\
\hline & $1^{s t}$ & $3^{r d}$ & $1^{\text {st }}$ & $3^{\text {rd }}$ \\
\hline $\begin{array}{l}\text { (1) Reached at } \\
\text { the appropriate DCP }\end{array}$ & 0.6 & 0.68 & 0.45 & 0.67 \\
\hline (2) Parking succeeded & 0.1 & 0.53 & 0.1 & 0.65 \\
\hline $\begin{array}{l}\text { (3) Parking succeeded from } \\
\text { the appropriate DCP }\end{array}$ & 0.17 & 0.69 & 0.11 & 0.71 \\
\hline $\begin{array}{l}\text { (4) Parking succeeded from } \\
\text { the inappropriate DCP }\end{array}$ & 0 & 0.17 & 0.09 & 0.5 \\
\hline
\end{tabular}

in the analysis of the experiment in Section III. The sum of the normalized translation/orientation errors in the parking position and the time taken to park is defined as the parking score. Figure 10 shows examples of (a) successful and (b) failed parking, and the normalized parking scores, and Fig. 11 shows the averaged parking score for both groups in $1^{\text {st }}$ and $3^{\text {rd }}$ sets. The results reveal significant difference for a Mann-Whitney's U-test $(p<0.01$, double-ended) between the two groups in the $3^{\text {rd }}$ set, whereas no difference was observed in the $1^{\text {st }}$ set.

\subsection{Relationship between Direction-Change Point and Parking Result}

Reverse parking consists of two different tasks: driving forward from the initial position to the directionchange point (DCP), and driving backward from the DCP to the parking position. However, due to the nonholonomic characteristics of the vehicle and the limitations on the number of direction changes, the difficulty of driving backward is dependent on the DCP. Therefore, to evaluate the effect of the proposed assistance method, which is focused on supporting 


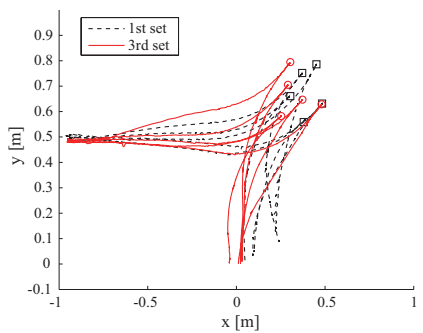

(a)

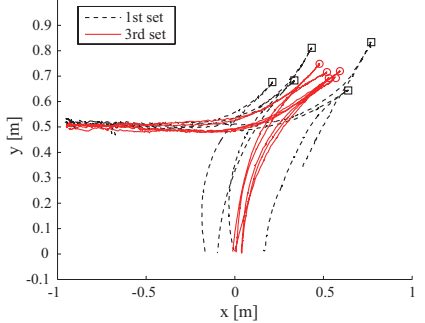

(b)
Fig. 12. Examples of parking trajectories of the subjects in Group A (non-assisted). Black dotted lines represent the trajectories for the $1 \mathrm{st}$ set, and red lines represent the trajectories for the $3^{\text {rd }}$ set. The parking trajectories of the assisted subjects tended to converge.

the driving after the DCP, the parking results should be considered by taking into consideration the adequacy of the DCP. Table 5 describes the relationship between the rate of successful parking and the DCP, where the appropriate DCP is defined as the point for which the optimal path can be derived using the pathplanning method explained in Section 4.1. A parking trial accomplished with less than $0.1[\mathrm{~m}]$ translation error and less than 0.1 [rad] orientation error is considered successful. From this table, (1) the percentage of parking from an appropriate DCP, (2) the rate of successful parking and, (3) the rate of successful parking from the appropriate DCP increased between $1^{\text {st }}$ and $3^{\text {rd }}$ sets. However, regarding (4) the rate of successful parking from an inappropriate DCP, the subjects assisted by the system had $50 \%$ rate of parking success, which was higher than that of the non-assisted subjects $(17 \%)$.

\subsection{Analysis of Direction-Change Point Variance}

To investigate the effect of the proposed assistance method on learning the appropriate DCP, we analyzed the variance of the DCP distribution and its change between trials. Because the $1^{\text {st }}$ principal component of the distribution represents the maximum variance, it can be considered as a quantitative feature of the DCP distribution. Figure 13 is a comparison of the average DCP distribution variances for each group. The results shows that the variance for the assisted subjects decreased in the $3^{r d}$ set, with more than $95 \%$ significance for a double-ended Mann-Whitney's Utest. In contrast, there is no significant improvement in the group of non-assisted subjects, although the average variance increased slightly.

\subsection{Discussion}

First of all, there were no significant differences between the reverse parking skills of the two groups at the beginning of the experiment according to Fig.

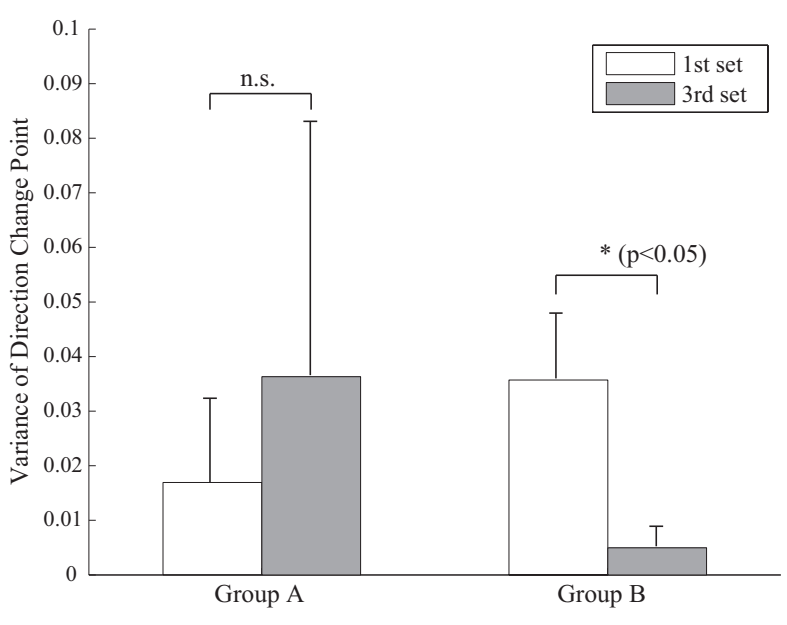

Fig. 13. Absolute variance of the direction-change point. The variance for Group B decreased significantly $(p<0.05$, Mann-Whitney's U-test)

11, which shows the parking score, a quantification of the parking accuracy and time taken. However, in the $3^{\text {rd }}$ set, the average parking score of the assisted subjects increased significantly compared to the control subjects. This means that the assistance, which was activated in the $2^{\text {nd }}$ set of Group B, improved the skill of the subjects for reverse parking.

Regarding the relationship between the DCP and the parking result, the rate of successful parking from an inappropriate DCP for Group B improved significantly. This indicates that the difference in the parking scores of two groups (Fig. 11) was caused by the improved reverse driving skill after the DCP. In other words, reaching an appropriate DCP does not always guarantee successful parking, and parking in an appropriate position from an inappropriate DCP is also possible. Therefore, the reverse driving skill of Group B, which was improved by the assistance system, contributes to parking with high scores. This can be considered as a reason for the difference between the rate of successful parking and the parking scores.

Conversely, because the rate of reaching appropriate DCPs for both groups converged to almost the same value in the $3^{\text {rd }}$ set, we could not confirm the direct effect of the proposed method on learning an appropriate DCP. This is understandable because the driver could choose any DCP, and the system did not suggest a DCP in thie experiment. However, the absolute variance of the DCP for Group B decreased significantly, whereas that for Group A did not. During the experiment, we observed that the subjects focused on seeking better DCPs even though the previous parking trial failed as a result of inappropriate operation after the DCP. In other words, those subjects did not realize the true cause of failure and tried to improve by a wrong approach. 
This was why the DCP variance for Group A did not converge in the $3^{\text {rd }}$ set, although there was a higher rate of choosing appropriate DCPs $(60 \%)$ in the $1^{\text {st }}$ set. Conversely, because the subjects in Group B could park successfully using the assistance system, they did not need further trials to find a new DCP and could focus on learning the appropriate operation demonstrated by the assistance. Moreover, the LED feedback when the DCP was inappropriate during the $2^{\text {nd }}$ set facilitated DCP exploration for the Group B subjects.

Finally, we discuss the dependencies of the reverse parking skill acquired by means of the proposed assistance system on the specific environment (in this case, the scaled car simulator). There were no objects that could be used as landmarks to determine the position and orientation of the vehicle, other than the lines and road cones that indicated the target parking space in the experimental environment. This means that the subjects learned the appropriate DCP and driving operations using the estimated relative position with respect to the target parking space, which was observed through the mirrors and windows of the simulator. In this sense, the driving skill acquired by the subjects was independent on the scaled car simulator and can therefore be used for more general situations of reverse parking.

\section{CONCLUSION}

In this paper, we introduced a haptic-interventionbased driving assistance system for improving the reverse parking skill of drivers. The fundamental concept underlying the proposed method is the enhancement of the cognitive-motor learning process of the driver by means of a haptic assistance coordinated by the present state of the vehicle. To design the assistance method, we first used a real car to conduct a measurement experiment to characterize human driving, and then analyzed the relationship between the parking score and the identified driving parameters. Based on the acquired knowledge about the characteristics of successful driving such as operating with acceleration/deceleration and minimizing the total amount of operation, we developed a scaled car simulator that comprised a $1 / 10$ scaled robot car and an omnidirectional camera, which we used as a simulation environment for assessing the proposed method. We used subjects to conduct experiments in the simulator and confirmed that the parking scores of the assisted subjects were significantly better than those of the control group subjects. In addition, the variance of the direction-change point of the assisted subjects decreased compared to that of the control group subjects. This indicates that our proposed method might also be used for learning an appropriate DCP.

In a future work, we intend to implement a mechanism for configuring the assistance parameters (such as the strength) that correspond to the gain parameters and the timing of the launch of the assistance, with adaptation to individual subjects. Wwe would also like to adapt the assistance method to pedal operation and apply it to other driving processes other than reverse parking using cognitive and motion assistance technologies.

\section{REFERENCES}

[1] J. Sil and T. Srikanthan, "A hybrid approach to designing an autonomous driving alert system using geometrical features and gray level information of face images," in IEEE Region 5, 2003 Annual Technical Conference, 2003, pp. 33-38.

[2] K. Kojima, A. Sato, F. Taya, Y. Kameda, and Y. Ohta, "Naviview: visual assistance by virtual mirrors at blind intersection," in Intelligent Transportation Systems, 2005. Proceedings. 2005 IEEE, 2005, pp. 592-597.

[3] M. Tonnis, C. Lange, and G. Klinker, "Visual longitudinal and lateral driving assistance in the head-up display of cars," in Mixed and Augmented Reality, 2007. ISMAR 2007. 6th IEEE and ACM International Symposium on, 2007, pp. 91-94.

[4] P. Griffiths and R. Gillespie, "Sharing control between humans and automation using haptic interface: primary and secondary task performance benefits," Human Factors, vol. 47, no. 3, pp. 574-590, 2005.

[5] J. Navarro, F. Mars, and M. S. Young, "Lateral control assistance in car driving: classification, review and future prospects," Intelligent Transport Systems, IET, vol. 5, no. 3, pp. 207-220, 2011.

[6] L. Saleh, P. Chevrel, F. Mars, J. Lafay, and F. Claveau, "Humanlike cybernetic driver model for lane keeping," World Congress, vol. 18, no. 1, pp. 4368-4373, 2011.

[7] L. Saleh, P. Chevrel, F. Claveau, J.-F. Lafay, and F. Mars, "Shared steering control between a driver and an automation: Stability in the presence of driver behavior uncertainty," Intelligent Transportation Systems, IEEE Transactions on, vol. 14, no. 2, pp. 974-983, 2013.

[8] M. Deroo, J.-M. Hoc, and F. Mars, "Effect of strength and direction of haptic cueing on steering control during near lane departure," Transportation Research Part F: Traffic Psychology and Behaviour, vol. 16, no. 0, pp. 92 - 103, 2013.

[9] M. Mulder, D. Abbink, and E. Boer, "The effect of haptic guidance on curve negotiation behavior of young, experienced drivers," in Systems, Man and Cybernetics, 2008. SMC 2008. IEEE International Conference on, 2008, pp. 804-809.

[10] N. Kuge and T. Yamamura, "Study on driver's car following abilities based on an active haptic support function," $S A E$ Transactions Journal of Passenger Cars - Electronic and Electrical Systems, vol. 115, no. 7, pp. 157-169, 2006.

[11] S. Azzi, G. Reymond, F. Merienne, and A. Kemeny, "Ecodriving performance assesment with in-car visual and haptic feedback assistance," Journal of Computing and Infomation Science in Engineering, vol. 11, no. 4, 2011.

[12] J.-M. Hoc, M. S. Young, and J.-M. Blosseville, "Cooperation between drivers and automation: implications for safety," Theoretical Issues in Ergonomics Science, vol. 10, no. 2, pp. 135160, 2009.

[13] Y. Makino, Y. Tanaka, T. Endo, and Y. Kawabata, "Development of intelligent parking assist," Journal of Society of Automotive Engineers of Japan, vol. 60, no. 10, pp. 47-52, 2006.

[14] K. Sakai, "Development of smart parking assist system," Journal of Society of Automotive Engineers of Japan, vol. 63, no. 12, pp. 60-64, 2009.

[15] M. R. Endsley and E. O. Kiris, "The out-of-the-loop performance problem and level of control in automation," Human Factors: The Journal of the Human Factors and Ergonomics Society, vol. 37, no. 2, pp. 381-394, 1995. [Online]. Available: http://hfs.sagepub.com/content/37/2/381.abstract

[16] T. Inagaki, "Design of humanmachine interactions in light of domain-dependence of human-centered automation," Cognition, Technology and Work, vol. 8, no. 3, pp. 161-167, 2006. [Online]. Available: http://dx.doi.org/10.1007/s10111006-0034-z 
[17] W. Night, "Proceed with caution toward the self-driving car," MIT Technology Review, 2013.

[18] M. Aisen, s. H. Kreb, N. Hogan, F. McDowell, and B. Volpe, "The effect of robot-assisted therapy and rehabilitative training on motor recovery following stroke," Archives of Neurology, vol. 54, no. 4, pp. 443-446, 1997. [Online]. Available: + http://dx.doi.org/10.1001/archneur.1997.00550160075019

[19] X.-D. Yang, W. Bischof, and P. Boulanger, "Validating the performance of haptic motor skill training," in Haptic interfaces for virtual environment and teleoperator systems, 2008. haptics 2008. symposium on, 2008, pp. 129-135.

[20] D. Powell and M. O'Malley, "The task-dependent efficacy of shared-control haptic guidance paradigms," Haptics, IEEE Transactions on, vol. 5, no. 3, pp. 208-219, 2012.

[21] E. H. van Asseldonk, M. Wessels, A. H. Stienen, F. C. van der Helm, and H. van der Kooij, "Influence of haptic guidance in learning a novel visuomotor task," Journal of Physiology-Paris, vol. 103, no. 3?5, pp. 276 - 285, 2009, ¡ce:title ¿Neurorobotics; /ce:title ${ }_{i}$.

[22] R. Okita, M. Shibakawa, A. Tsukuda, Y. Ishii, T. Ito, and N. Kawahara, "Outline of intelligent drive master(i-dm)," Mazda Technical Review, vol. 29, 2011.

[23] M. Yamakado, J. Takahashi, S. Saito, and M. Abe, "New vehicle dynamics control concept "g-vectoring" for enhancement of safe driving," Hitachi Review, no. 10, pp. 784-787, 2012.

[24] T. Ohtsuka and H. A. Fujii, "Real-time optimization algorithm for nonlinear receding-horizon control," Automatica, vol. 33, no. 6, pp. 1147 - 1154, 1997.

[25] T. Kobayashi and S. Majima, "Real-time optimization control for parking a vehicle automatically," in Vehicle Electronics Conference, 2001. IVEC 2001. Proceedings of the IEEE International, 2001, pp. 97-102.

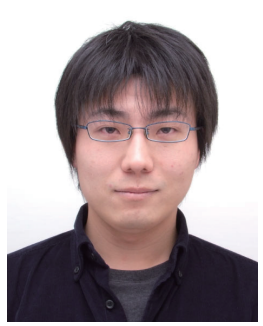

Masakazu Hirokawa receided Engineer's degree from College of Engineering Systems, University of Tsukuba, Japan in 2008 and M.Eng from Graduate School of Systems and Information Engineering, University of Tsukuba in 2011 . He is currently a Ph.D candidate in Graduate School of Systems and Information Engineering, University of Tsukuba, Japan and a Research Fellow of the Japan Society for the Promotion of Science.

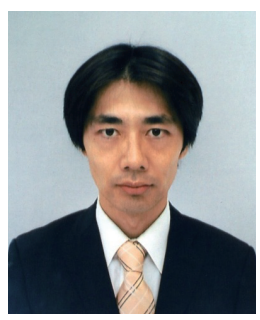

Naohisa Uesugi received Master degree of Engineering from University of Tsukuba, Japan, in 2001, and Master degree of Business Administration from Yokohama National University, Japan, in 2010. He has been an engineer at Mazda Motor Corporation since 2001. He is currently in charge of a series of research on" Kansei" engineering.

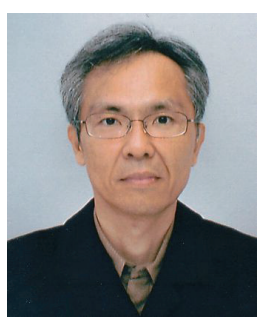

Satoru Furugori received bachelor degree of engineering from the department of administration engineering of Keio university, in 1985. He joined Mazda in 1985. In recent years, he has been working in research areas such as human factors in automobile, system design of future mobility as a senior specialist in the technical research center.

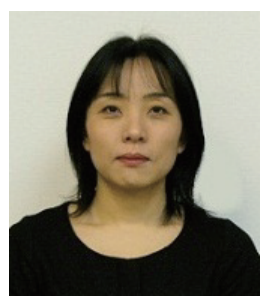

Tomoko Kitagawa is a technical specialist in Technical Research Center of Mazda Motor Corporation since 1992. She is specialized in data analysis such as text mining. I $n$ recent years, she is also working in the area of Kansei " engineering, i.e., emotional engineering, in automobile.

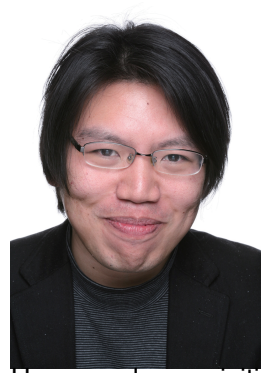

Kenji Suzuki received the B.S. degree in Physics, M.E. and Ph.D. degrees in Pure and Applied Physics from Waseda University, Tokyo, Japan, in 1997, 2000 and 2003, respectively. He is currently an Associate Professor at the Center for Cybernics Research, and also the Principal Investigator of Artificial Intelligence Laboratory, University of Tsukuba, Japan. From 2002 to 2005, he was a Research Associate at the Dept. of Applied Physics, Waseda University, Japan. He was also a visiting researcher at the Laboratory of Physiology of Perception and Action, College de France, in 2009, and the Laboratory of Musical Information, University of Genoa, Italy in 1997. His research interests include Assistive and Rehabilitation Robotics, Cognitive Robotics, Biosignal Processing, and Computational Behavior Science. 